IPPP-03-12

DCPT-03-24

hep-ph/0303234

\title{
A Future Linear Collider with Polarised Beams Searches for New Physics ${ }^{1}$
}

\author{
Gudrid Moortgat-Pick ${ }^{2}$ \\ IPPP, University of Durham, Durham DH1 3LE, U.K. ${ }^{3}$
}

\begin{abstract}
There exists a world--wide consensus for a future $e^{+} e^{-}$Linear Collider in the energy range between $\sqrt{s}=500-1000 \mathrm{GeV}$ as the next large facility in HEP. The Linear Collider has a large physics potential for the discovery of new physics beyond the Standard Model and for precision studies of the Standard Model itself. It is well suited to complement and extend the physics program of the LHC. The use of polarised beams at a Linear Collider will be one of the powerful tools. In this paper some highlights of searches for physics beyond the Standard Model at a future Linear Collider with polarised $e^{-}$and $e^{+}$beams are summarised.
\end{abstract}

\section{Beam Polarisation at a Linear Collider}

The next future large experiment in high energy physics will most probably be a future Linear Collider (LC) in the energy range between LEP and $\mathrm{O}(1 \mathrm{TeV})$. The existing world-wide proposals are designed with high luminosity of about $\mathcal{L}=3.4 \cdot 10^{34} \mathrm{~cm}^{-2} \mathrm{~s}^{-1}$ at $\sqrt{s}=500 \mathrm{GeV}$ and $\mathcal{L}=$ $5.8 \cdot 10^{34} \mathrm{~cm}^{-2} \mathrm{~s}^{-1}$ at $\sqrt{s}=800 \mathrm{GeV}$, see e.g. 11. A LC will not only be well suited to complement 2 but also to extend the physics program of the Hadron Colliders, the Tevatron and the future Large Hadron Collider (LHC).

A LC has a large potential for the discovery of new particles and is due to its clear signatures - very well suited for the precise analysis of new

\footnotetext{
${ }^{1}$ Invited plenary talk given at 15th International Spin Physics Symposium, Sept. 2002, BNL, Brookhaven, USA.

${ }^{2}$ email: g.a.moortgat-pick@durham.ac.uk.

${ }^{3}$ Address before January 2003: DESY, Deutsches Elektronen-Synchrotron, D-22603 Hamburg, Germany.
} 
physics (NP) as well as of the Standard Model (SM) 11. Providing precision studies in this energy range, in particular in the electro-weak sector, even small traces of physics beyond the SM might be found, even if high scale particles of a NP might not be directly produced at the LHC or the first phase of a LC. For these studies the GigaZ option of the LC, i.e. running with very high luminosity at the $Z$ and the $W W$ threshold, is decisive.

An important tool of a $\mathrm{LC}$ is the use of polarised beams. In the following we will summarise some highlights of searches and analyses of new physics with the help of polarised beams [1, 3, 4].

Already in the base line design it is foreseen to use electron beams polarised to around $80 \%$ via a strained photocathode technology [1 similar to those at the SLC where in the last year of running 1994/95 $P_{e^{-}}=$ $(77.34 \pm 0.61) \%$ ( $[3$ and references therein) was reached. In order to generate also polarised positrons the use of a helical undulator is favoured producing polarised photons which generate via pair production positrons with a designed polarisation degree of about $40 \%$ (with full intensity of the $e^{+}$beam) up to $60 \%$ (with probably about $55 \%$ intensity) [5]. There already exists a world-wide collaboration supporting the activities to get a prototype for a polarised positron source at the $50 \mathrm{GeV}$ Final Focus Test Beam at SLAC 6 .

It is foreseen to measure the polarisation with Compton polarimetry and it is assumed that one could reach even an accuracy better than $\Delta\left(P_{e^{ \pm}}\right)<$ $0.5 \%$ [7. The simultaneous use of $\mathrm{M} \phi$ ller polarimetry will also be studied at the LC 8]. However, the reachable accuracy with Compton and M $\phi$ ller polarimetry will not be sufficient for the high precision tests at GigaZ. For this purpose one uses an alternative Blondel Scheme [9], see next section, where one expresses the polarisation via polarised cross sections. Therefore one can avoid absolute measurements of polarisation and uses polarimetry only for relative measurements.

After a short introduction into the physics of beam polarisation we begin our summary with high precision studies of the SM as a motivation for new physics searches.

\section{$1.1 \quad$ Introductory remarks}

Within the Standard Model (SM) only $(V-A)$ couplings happen in the s-channel and therefore the configurations $L R$ and $R L$ are possible for the $e^{-} e^{+}$helicities. That means that once the $e^{-}$polarisation is chosen also the $e^{+}$polarisation is fixed. For these processes an additional simultaneous positron polarisation leads to an enhancement (or suppression) of the frac- 


\begin{tabular}{|l||c|c|c|c||c|c|}
\hline & RL & LR & RR & LL & $P_{\text {eff }}$ & $\mathcal{L}_{\text {eff }} / \mathcal{L}$ \\
\hline $\begin{array}{l}P_{e^{-}}=0, \\
P_{e^{+}}=0\end{array}$ & 0.25 & 0.25 & 0.25 & 0.25 & 0. & 0.5 \\
\hline $\begin{array}{l}P_{e^{-}}=-1, \\
P_{e^{+}}=0\end{array}$ & 0 & 0.5 & 0 & 0.5 & -1 & 0.5 \\
\hline $\begin{array}{l}P_{e^{-}}=-0.8, \\
P_{e^{+}}=0\end{array}$ & 0.05 & 0.45 & 0.05 & 0.45 & -0.8 & 0.5 \\
\hline $\begin{array}{l}P_{e^{-}}=-0.8, \\
P_{e^{+}}=+0.6\end{array}$ & 0.02 & 0.72 & 0.08 & 0.18 & -0.95 & 0.74 \\
\hline
\end{tabular}

Table 1: Fraction of colliding particles $\left(\mathcal{L}_{\text {eff }} / \mathcal{L}\right)$ and the effective polarisation $\left(P_{\text {eff }}\right)$ for different beam polarisation configurations, which are characteristic for $(\mathrm{V}-\mathrm{A})$ processes in the $\mathrm{s}-$ channel [10].

tion of the colliding particles, which is expressed by the effective luminosity

$$
\mathcal{L}_{\text {eff }} / \mathcal{L}:=\frac{1}{2}\left(1-P_{e^{-}} P_{e^{+}}\right)
$$

and of the effective polarisation

$$
P_{e f f}:=\left(P_{e^{-}}-P_{e^{+}}\right) /\left(1-P_{e^{-}} P_{e^{+}}\right) .
$$

In Table 1 we list $\mathcal{L}_{\text {eff }}$ and $P_{\text {eff }}$ for some characteristic values of $P_{e^{-}}$and $P_{e^{+}}$. One can see that even with a completely polarised $e^{-}$beam the fraction of colliding particles is not enhanced, however, with simultaneously polarised positrons this fraction will be enhanced.

It is well-known that with suitably polarised beams one can suppress background processes, e.g. the dominating SM backgrounds $e^{+} e^{-} \rightarrow W^{+} W^{-}$ and $Z Z$. Some scaling factors $\sigma^{\text {pol }} / \sigma^{\text {unpol }}$ for these processes are given in Table 2

Moreover, beyond the SM there are also coupling structures in the schannel possible where also the configurations $L L$ and $R R$ could lead to strong signals. Simultaneous polarisation of both beams would lead therefore to fast and easy diagnostics and we will give one example.

These given 'rules' are not generally valid for t-channel exchanges since in that case the helicity of the incoming $e^{-}$is only coupled to the outgoing particle at the vertex and not to the incoming $e^{+}$. This can be easily seen when studying the well-known Bhabha background. For small energies the s-channel with its $L R$ and $R L$ coupling characteristics dominates. However, 


\begin{tabular}{|c|c|c|}
\hline$\left(P_{e^{-}}=\mp 80 \%, P_{e^{+}}=0, \pm 60 \%\right)$ & $e^{+} e^{-} \rightarrow W^{+} W^{-}$ & $e^{+} e^{-} \rightarrow Z Z$ \\
\hline$(+0)$ & 0.2 & 0.76 \\
$(-0)$ & 1.8 & 1.25 \\
\hline$(+-)$ & 0.1 & 1.05 \\
$(-+)$ & 2.85 & 1.91 \\
\hline
\end{tabular}

Table 2: Scaling factors $\sigma^{\text {pol }} / \sigma^{\text {unpol }}$ for the dominating SM background processes $e^{+} e^{-} \rightarrow W^{+} W^{-}$and $Z Z$ for different configurations of beam polarisation [3].

for higher energies, also $L L$ coupling is possible via the $\nu$-exchange in the tchannel. Another example, where the use of both beams polarised is obvious, is the single $W$ background since with $e^{-}$polarisation only the $W^{-}$signal can be suppressed. For the corresponding signal from $W^{+}$the polarisation of $e^{+}$is needed.

\section{Electroweak high precision analyses of the SM}

Electroweak precision tests with an unprecedented accuracy - at high energies as well as at the $Z$ resonance and the $W W$ threshold - would allow to see hints for new physics, even if new particles are not directly produced. In the following section we list some examples for these high precision measurements at a LC, e.g. the measurement of triple gauge couplings. After that we will have a look at the additional prospects of GigaZ which is planned as an upgrade.

\subsection{Anomalous couplings in $e^{+} e^{-} \rightarrow W^{+} W^{-}$}

In order to test the SM with high precision one can carefully study triple gauge boson couplings, which are generally parametrised in an effective Lagrangian e.g. by the $\mathrm{C}-, \mathrm{P}-$ conserving couplings $g_{1}^{V}, \kappa_{V}, \lambda_{V}$ with $V=\gamma, Z$. In the SM at tree level the couplings have to be $g_{1}^{V}=1=\kappa_{V}$, while $\lambda_{V}$ are identical to zero.

These couplings can be determined by measuring the angular distribution and polarisation of the $W^{ \pm}$'s. Simultaneously fitting of all couplings results in a strong correlation between the $\gamma-$ and $Z$-couplings. It turns out that the polarisation of the beams is very powerful for separating these couplings: e.g. the polarisation of $P_{e^{-}}= \pm 80 \%$ (together with $P_{e^{+}}=\mp 60 \%$ ) 


\begin{tabular}{|cccccr|}
\hline error $\left[10^{-4}\right]:$ & $\Delta g_{Z}^{1}$ & $\Delta \kappa_{\gamma}$ & $\lambda_{\gamma}$ & $\Delta \kappa_{Z}$ & $\lambda_{Z}$ \\
\hline \hline \multicolumn{7}{|c|}{ unpolarised beams } \\
\hline$\sqrt{s}=500 \mathrm{GeV}$ & 38.1 & 4.8 & 12.1 & 8.7 & 11.5 \\
$\sqrt{s}=800 \mathrm{GeV}$ & 39.0 & 2.6 & 5.2 & 4.9 & 5.1 \\
\hline \multicolumn{6}{|c|}{ only electron beam polarised, $\left|P_{e^{-}}\right|=80 \%$} \\
\hline$\sqrt{s}=500 \mathrm{GeV}$ & 24.8 & 4.1 & 8.2 & 5.0 & 8.9 \\
$\sqrt{s}=800 \mathrm{GeV}$ & 21.9 & 2.2 & 5.0 & 2.9 & 4.7 \\
\hline both beams polarised, $\left|P_{e^{-}}\right|=80 \%,\left|P_{e^{+}}\right|=60 \%$ \\
\hline$\sqrt{s}=500 \mathrm{GeV}$ & 15.5 & 3.3 & 5.9 & 3.2 & 6.7 \\
$\sqrt{s}=800 \mathrm{GeV}$ & 12.6 & 1.9 & 3.3 & 1.9 & 3.0 \\
\hline
\end{tabular}

Table 3: Sensitivity for anomalous triple gauge couplings with different configurations of beam polarisation [1].

improves the sensitivity up to a factor 1.8 (2.5), see Table 3 [1]. 11.

\subsection{Transversely polarised beams in $e^{+} e^{-} \rightarrow W^{+} W^{-}$}

Another promising possibility to study the origin of electroweak symmetry breaking is the use of transversely polarised $e^{+} e^{-}$beams which projects out $W_{L}^{+} W_{L}^{-}$[12]. The asymmetry with respect to the azimuthal angle of this process focusses on the $L L$ mode. This asymmetry is very pronounced at high energies reaching about $10 \%$. The advantage of this observable is that at high energies this asymmetry peaks at larger angles and not in beam direction where the analysis might be difficult. One has to note, however, that for the use of transverse beams the polarisation of both beams is needed. The effect does not occur if only one beam is polarised since the cross section is given by:

$$
\sigma=\left(1-P_{e^{-}}^{L} P_{e^{+}}^{L}\right) \sigma_{u n p}+\left(P_{e^{-}}^{L}-P_{e^{+}}^{L}\right) \sigma_{p o l}^{L}+P_{e^{-}}^{T} P_{e^{+}}^{T} \sigma_{p o l}^{T} .
$$

\subsection{GigaZ}

At the GigaZ option $e^{+} e^{-} \rightarrow Z \rightarrow f \bar{f}$ is studied and the effective electroweak leptonic mixing angle can be measured via the left-right asymmetry

$$
A_{L R}=\frac{2\left(1-4 \sin ^{2} \Theta_{e f f}^{\ell}\right)}{1+\left(1-4 \sin ^{2} \Theta_{e f f}^{\ell}\right)^{2}}
$$

of this process. Since one gets only a gain in statistical power if the error due to the polarisation measurement $\Delta A_{L R}(\mathrm{pol})$ is smaller than the statistical 
error $\Delta A_{L R}($ stat $)$ one has to know $P_{e^{ \pm}}$extremely accurately. Up to now even $\Delta P_{e^{ \pm}}<0.5 \%$ would not be sufficient. Therefore one uses an alternative Blondel Scheme [1], 1] and expresses $A_{L R}$ via polarised rates:

$$
A_{L R}=\sqrt{\frac{\left(\sigma^{++}+\sigma^{+-}-\sigma^{-+}-\sigma^{--}\right)\left(-\sigma^{++}+\sigma^{+-}-\sigma^{-+}+\sigma^{--}\right)}{\left(\sigma^{++}+\sigma^{+-}+\sigma^{-+}+\sigma^{--}\right)\left(-\sigma^{++}+\sigma^{+-}+\sigma^{-+}-\sigma^{--}\right)}}
$$

With this method, polarimetry has to be used only for calibration and one can reach a spectacular accuracy for the electroweak observables, see Table 4 11. The polarisation of the positron beam is absolutely needed but already a polarisation of about $P_{e^{+}}=|40 \%|$ would be sufficient, see Fig. 1a, to measure these observables with an unprecedented accuracy.

As an example of the potential of the GigaZ $\sin ^{2} \theta_{\text {eff }}$ measurement, Fig. 10 [13] compares the present experimental accuracy on $\sin ^{2} \theta_{\text {eff }}$ and $M_{W}$ from LEP/SLD/Tevatron and the prospective accuracy from the LHC and from a LC without GigaZ option with the predictions of the SM and the MSSM. With GigaZ a very sensitive test of the theory will be possible.

a)

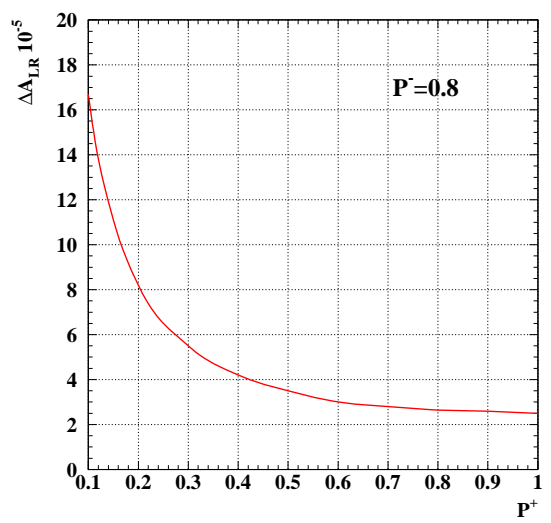

b)

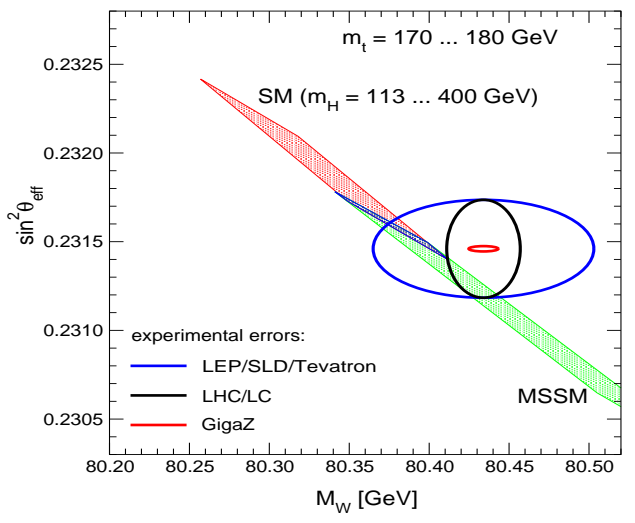

Figure 1: a) Test of Electroweak Theory: The statistical error on the leftright asymmetry $A_{L R}$ of $e^{+} e^{-} \rightarrow Z \rightarrow \ell \bar{\ell}$ at GigaZ as a function of the positron polarisation $P_{e^{+}}$for fixed electron polarisation $P_{e^{-}}= \pm 80 \%$ [11]. In b) The theoretical prediction for the relation between $\sin ^{2} \theta e f f$ and $M_{W}$ in the SM for Higgs boson masses in the intermediate range is compared to the experimental accuracies at LEP 2/Tevatron (Run IIA), LHC/LC and GigaZ [13.

It should also be mentioned that with the help of polarised beams a LC could be sensitive to electroweak dipole form factors. They have been analysed 


\begin{tabular}{c|cccc} 
& LEP2/Tev. & Tev./LHC & LC & GigaZ/WW \\
\hline$M_{W}$ & $34 \mathrm{MeV}$ & $15 \mathrm{MeV}$ & $15 \mathrm{MeV}$ & $6 \mathrm{MeV}$ \\
$\sin ^{2} \theta_{\text {eff }}$ & 0.00017 & 0.00017 & 0.00017 & 0.00001 \\
$m_{t}$ & $5 \mathrm{GeV}$ & $2 \mathrm{GeV}$ & $0.2 \mathrm{GeV}$ & $0.2 \mathrm{GeV}$ \\
$m_{h}$ & - & $0.2 \mathrm{GeV}$ & $0.05 \mathrm{GeV}$ & $0.05 \mathrm{GeV}$
\end{tabular}

Table 4: Sensitivity to electroweak observables at different colliders in comparison [13].

in [14 with regard to $\mathrm{CP}$ violation of the $\tau$ lepton and via $\mathrm{CP}$-odd triple product correlations. Sensitivity bounds for the real and imaginary parts of these form factors have been set up to $O\left(10^{-19}\right)$ ecm.

In the same context one should not forget that also for searches of heavy gauge bosons, as e.g. for the $Z^{\prime}$, the use of polarised beams enhances the discovery range. Also for contact interactions the sensitivity can be enhanced significantly [3, 15].

\section{Beyond the Standard Model}

\subsection{Supersymmetry}

Supersymmetry is widely regarded as one of the best motivated extensions of the SM. However, since the SM particles and their SUSY partners are not mass degenerate, SUSY has to be broken, which leads even for its minimal version, the Minimal Supersymmetric Standard Model (MSSM), to about 105 free new parameters. In specific scenarios of SUSY breaking one can end with only a few parameters: 5 in mSUGRA, 4 in AMSB and 5 in GMSB. However, one should note that one of the most favoured motivation for SUSY - the unification of the gauge couplings - is consistent within the general MSSM independently of the large number of new parameters.

In order to exactly pin down the structure of the underlying model it is therefore unavoidable to extract the parameters without assuming a particular breaking scheme. Since the LC with its extremely clear signatures provides a measurement of the particle masses up to $O(100) \mathrm{MeV}$, of the rates and branching ratios at the \% level, the LC is well suited for revealing the underlying structure of the model. Different step-by-step procedures have been worked out to determine the general MSSM parameters and to test fundamental SUSY assumptions as e.g. the equality of quantum numbers or 
of couplings of the particles and their SUSY partners as model independent as possible. It turns out that the use of polarised beams plays a decisive role in this context.

\subsubsection{Stop mixing angle in $e^{+} e^{-} \rightarrow \tilde{t}_{1} \tilde{t}_{1}$}

As demonstrated in [1] the mass and the mixing angle of $\tilde{t}$ can be extracted with high precision via the study of polarised cross sections for light stop production. At a high luminosity LC and with $P\left(e^{-}\right)=80 \%$ and $P\left(e^{+}\right)=60 \%$ an accuracy of $\delta\left(m_{\tilde{t}_{1}}\right) \approx 0.8 \mathrm{GeV}$ and $\delta\left(\cos \theta_{\tilde{t}}\right) \approx 0.008$ could be reachable, see Fig. 2] [16]. Similar studies have been done for the $\tilde{\tau}$ sector [21].

\subsubsection{Quantum numbers in $e^{+} e^{-} \rightarrow \tilde{e}_{L, R}^{+} \tilde{e}_{L, R}^{-}$}

SUSY transformations associate chiral leptons to their scalar SUSY partners: $e_{L, R}^{-} \leftrightarrow \tilde{e}_{L, R}^{-}$and the antiparticles $e_{L, R}^{+} \leftrightarrow \tilde{e}_{R, L}^{+}$. In order to prove this association between scalar particles and chiral quantum numbers the use of polarised beams is necessary [18]. The process occurs via $\gamma$ and $Z$ exchange in the s-channel and via $\tilde{\chi}_{i}^{0}$ exchange in the t-channel. As already mentioned in the general introduction one has direct coupling between the SM particle and its scalar partner only in the t-channel. Therefore one has to project out the $\mathrm{t}$-channel exchange in order to test the association of chiral quantum numbers to the scalar SUSY partners.

With e.g. completely polarised $e_{L}^{-} e_{L}^{+}$only the pair $\tilde{e}_{L}^{-} \tilde{e}_{R}^{+}$contributes. Due to their $L, R$ coupling character $\tilde{e}_{L}, \tilde{e}_{R}$ can be discriminated via their decay characteristics and can be identified via their charge. One has to note that a polarised $e^{+}$beam is necessary. Even completely polarised $e^{-}$ would not be sufficient, since the s-channel exchange could not be switched off completely. However, even if only partially polarised beams of maximal $P_{e^{-}}=-80 \%$ and $P_{e^{+}}=-60 \%$ were available it could be sufficient to probe this association, since in this case the pair $\tilde{e}_{L}^{-} \tilde{e}_{R}^{+}$dominates by a factor of 3 in our example, Fig. [2], [18].

\subsubsection{Gaugino/higgsino sector}

The SUSY partners of the charged and neutral gauge bosons are the charginos $\tilde{\chi}_{1,2}^{ \pm}$and neutralinos $\tilde{\chi}_{1, \ldots, 4}^{0}$. Since SUSY is a broken the electroweak eigenstates mix and strategies have been worked out to determine the mixing angles via polarised rates in $e^{+} e^{-} \rightarrow \tilde{\chi}_{i}^{ \pm} \tilde{\chi}_{j}^{\mp}$ and $e^{+} e^{-} \rightarrow \tilde{\chi}_{i}^{0} \tilde{\chi}_{j}^{0}$ and to derive the underlying MSSM parameters ([19] and references therein). Even if only the lightest particles $\tilde{\chi}_{1}^{+} \tilde{\chi}_{1}^{-}, \tilde{\chi}_{1}^{0} \tilde{\chi}_{2}^{0}$ were accessible, it would be sufficient for 
a)

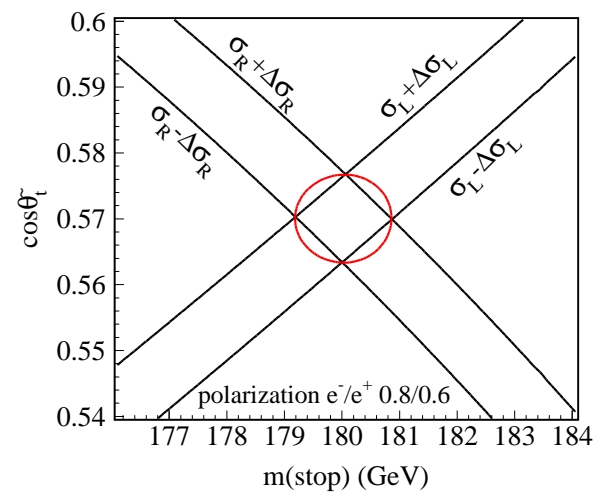

b)

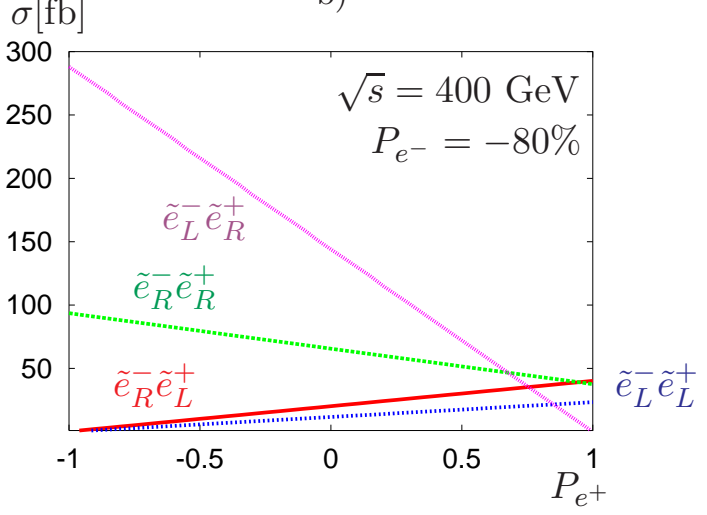

Figure 2: Test of selectron quantum numbers in $e^{+} e^{-} \rightarrow \tilde{e}_{L, R}^{+} \tilde{e}_{L, R}^{-}$with fixed electron polarisation $P\left(e^{-}\right)=-80 \%$ and variable positron polarisation $P\left(e^{+}\right)$. For $P\left(e^{-}\right)=-80 \%$ and $P\left(e^{+}\right)<0$ both pairs $\tilde{e}_{L}^{-} \tilde{e}_{R}^{+}$and $\tilde{e}_{R}^{-} \tilde{e}_{R}^{+}$still contribute. For $P\left(e^{+}\right)=-60 \%$ the pair $\tilde{e}_{L}^{-} \tilde{e}_{R}^{+}$dominates by more than a factor 3 [1, 3, 18.

determining the fundamental MSSM parameters $M_{1}, \Phi_{M_{1}}, M_{2}$ and $\mu, \Phi_{\mu}$, i.e. the $\mathrm{U}(1)$, the $\mathrm{SU}(2)$, and the higgsino mass parameters with its $\mathrm{CP}-$ violating phases. The ratio of the two Higgs vev's $\tan \beta=v_{2} / v_{1}$ can only be derived via this sector if $\tan \beta$ is not too large ([19] and references therein). In Fig. 3a, e.g., it is demonstrated, how to fix $\left|M_{1}\right|$ and $\Phi_{M_{1}}$ with polarised cross sections $\sigma\left(e^{+} e^{-} \rightarrow \tilde{\chi}_{1}^{0} \tilde{\chi}_{2}^{0}\right)$ and the light masses $m_{\tilde{\chi}_{1,2}^{0}}$. In this context the beam polarisation is needed in order to resolve ambiguities and to improve the statistics. Once the parameters are determined one can efficiently test whether the gauge couplings $g_{B e e}$ and $g_{W e e}$ are identical to the Yukawa couplings $g_{\tilde{B} e \tilde{e}}$ and $g_{\tilde{W} e \tilde{e}}$, respectively, by studying the polarised cross sections with a variable ratio of $g_{B e e} / g_{\tilde{B} e \tilde{e}}$ and $g_{W e e} / g_{\tilde{W} e \tilde{e}}$ and comparing it with experimental values [19], see Fig. 3b.

\subsubsection{The case of high $\tan \beta: \tau$ polarisation}

A crucial parameter is $\tan \beta$, but if $\tan \beta$ is high it will be very difficult to determine it very accurately. In case of high $\tan \beta>10$ the chargino and neutralino sector is insensitive to this parameter. But even in the Higgs sector the case $\tan \beta>10$ will lead to large uncertainties $\Delta(\tan \beta)>10 \%$ [20]. However, in many scenarios one could then determine $\tan \beta$ from another sector whose particles are relatively light: the $\tilde{\tau}$ sector [21]. 
a)

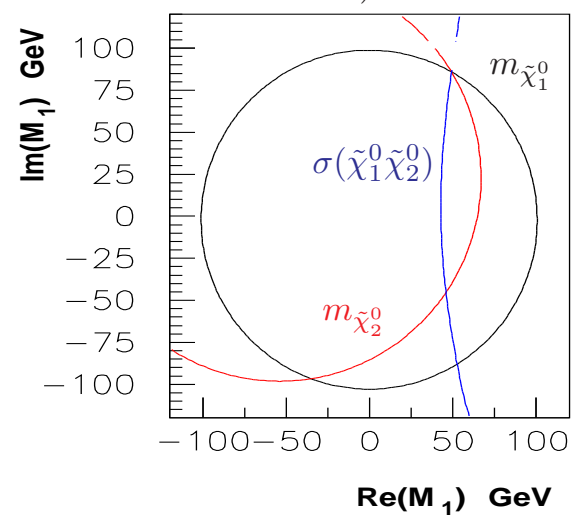

b)

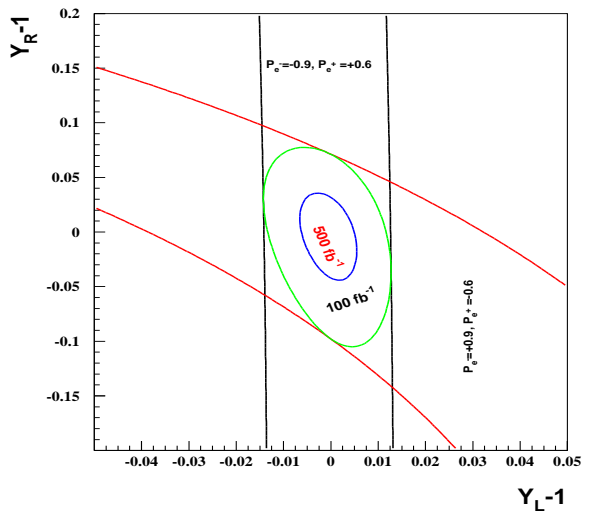

Figure 3: The contours of two neutralino masses $(1,2)$ and one neutralino production cross section $\sigma\left(e^{+} e^{-} \rightarrow \tilde{\chi}_{1}^{0} \tilde{\chi}_{2}^{0}\right)$ in the $\operatorname{Re}\left(M_{1}\right), \operatorname{Im}\left(M_{1}\right)$ plane; b) Contours of the cross sections $\sigma\left(e_{R}^{+} e_{L}^{-} \rightarrow \tilde{\chi}_{1}^{0} \tilde{\chi}_{2}^{0}\right)$ and $\sigma\left(e_{L}^{+} e_{R}^{-} \rightarrow \tilde{\chi}_{1}^{0} \tilde{\chi}_{2}^{0}\right)$ in the plane of the Yukawa couplings $g_{\tilde{W}}$ and $g_{\tilde{B}}$ normalised to the $\mathrm{SU}(2)$ and $\mathrm{U}(1)$ gauge couplings $g$ and $g^{\prime}\left\{Y_{L}=g_{\tilde{W}} / g, Y_{R}=g_{\tilde{B}} / g^{\prime}\right\}$ [19].

The polarisation of $\tau$ 's from $\tilde{\tau}_{i} \rightarrow \tau \tilde{\chi}_{j}^{0}$ is sensitive to $\tan \beta$ [17] and the $\tau$ polarisation can be rather accurately measured at a LC via e.g. the $\tau$ decays into $\pi$ 's, see Fig. 4a, 21. It has been discussed that in case of a sufficient higgsino admixture in the $\tilde{\chi}_{j}^{0}$ it is even possible to determine high $\tan \beta$ as well as $A_{\tau}$, without any assumptions on the SUSY breaking mechanism: after determining the $\tilde{\tau}$ mixing angle via polarised rates, preferable in the configuration $\sigma_{R L}$ due to $W W$ background suppression, one can determine $\tan \beta$ from the $\tau$ polarisation in the decay $\tilde{\tau}_{1} \rightarrow \tau \tilde{\chi}_{1}^{0}$, see Fig $4 \mathrm{~s}$. Even for high $\tan \beta \geq 20$ one can reach an accuracy of about $10 \%$.

\subsubsection{Extended SUSY models}

In case of e.g. R-parity violating SUSY non-standard couplings could occur which produce a scalar particle in the s-channel: $e^{+} e^{-} \rightarrow \tilde{\nu} \rightarrow e^{+} e^{-}$. The process gives a significant signal over the background. Since it requires both left-handed $e^{-}$and $e^{+}$beams - the $L L$ configuration- it can be eas- 

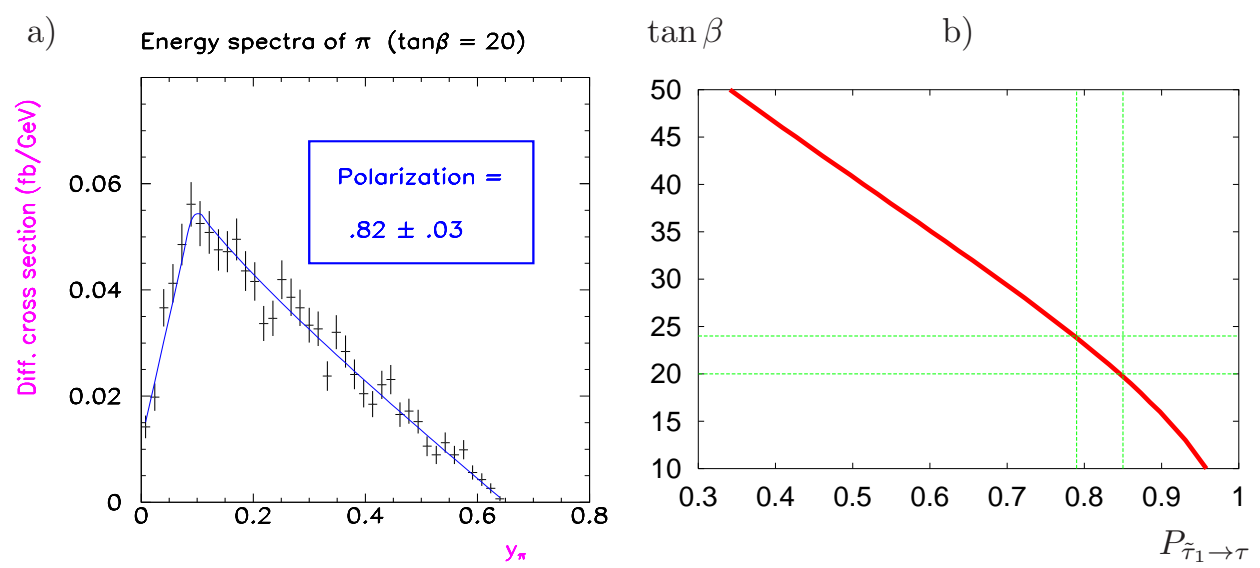

Figure 4: a) Determining the $\tau$ polarisation $P_{\tilde{\tau}_{1} \rightarrow \tau}$ from the pion energy distribution in the decay $\tilde{\tau}_{1} \rightarrow \tau \tilde{\chi}_{1}^{0} \rightarrow \nu_{\tau} \pi \tilde{\chi}_{1}^{0}$ leads to $\mathrm{b}$ ) an accurate determination of high $\tan \beta$ : e.g. $\tan \beta=20 \pm 2$ [21].

ily analysed and identified by the use of beam polarisation ([22, 3]): here simultaneously polarised beams enhance the signal by about a factor of 10 , see Table 5 .

One could also extend the MSSM without changing the gauge group, by introducing an additional Higgs singlet: it leads to the $(\mathrm{M}+1) \mathrm{SSM}$ with one additional neutralino. Since the mass spectra of the four light neutralinos could be similar to those in the MSSM in some parts of the $(\mathrm{M}+1) \mathrm{SSM} / \mathrm{MSSM}$ parameter space, a distinction between these models might be difficult via spectra and rates alone. However, polarisation effects might then indicate the different coupling structure in the $(\mathrm{M}+1) \mathrm{SSM}[23]$ and help disentangling the models, Fig. 5 .

\subsection{Large extra dimensions}

Another approach for physics beyond the SM, which could also resolve the hierachy problem, is the introduction of large extra dimensions. At a LC the process $e^{+} e^{-} \rightarrow \gamma G$ is promising and it has been worked out that with running on two different $\sqrt{s}$ one can determine the number of extra dimensions 


\begin{tabular}{|l||c|c|}
\hline & $\sigma\left(e^{+} e^{-} \rightarrow e^{+} e^{-}\right)$with & Bhabha-background \\
& $\sigma\left(e^{+} e^{-} \rightarrow \tilde{\nu} \rightarrow e^{+} e^{-}\right)$ & \\
\hline unpolarised & $7.17 \mathrm{pb}$ & $4.50 \mathrm{pb}$ \\
$P_{e^{-}}=-80 \%$ & $7.32 \mathrm{pb}$ & $4.63 \mathrm{pb}$ \\
$P_{e^{-}}=-80 \%, P_{e^{+}}=-60 \%$ & $8.66 \mathrm{pb}$ & $4.69 \mathrm{pb}$ \\
$P_{e^{-}}=-80 \%, P_{e^{+}}=+60 \%$ & $5.97 \mathrm{pb}$ & $4.58 \mathrm{pb}$ \\
\hline
\end{tabular}

Table 5: Sneutrino production in R-parity violating SUSY: Cross sections of $e^{+} e^{-} \rightarrow \tilde{\nu} \rightarrow e^{+} e^{-}$for unpolarised beams, $P_{e^{-}}=-80 \%$ and unpolarised positrons and $P_{e^{-}}=-80 \%, P_{e^{+}}=-60 \%$. The study was made for $m_{\tilde{\nu}}=$ $650 \mathrm{GeV}, \Gamma_{\tilde{\nu}}=1 \mathrm{GeV}$, an angle cut of $45^{0} \leq \theta \leq 135^{\circ}$ and the R-parity violating coupling $\lambda_{131}=0.05$ [22].

1. 24. The use of polarised beams in this context enlarges on one hand the sensitivity to the new scale $M_{*}$ and suppresses on the other hand the main background $e^{+} e^{-} \rightarrow \nu \nu \gamma$ significantly. The ratio $S / \sqrt{B}$ is enhanced by a factor of about $2.1(4.4)$ if $P_{e^{-}}=+80 \%$ (and $P_{e^{+}}=-60 \%$ ) is used.

\section{Summary}

A Linear Collider in the TeV range with its clean initial state of $e^{+} e^{-}$collisions is ideally suited for the search for new physics, for the determination of both Standard Model and non-standard couplings with high precision and for revealing the structure of the underlying model. The use of polarised beams plays a decisive role in this context. We have shown that simultaneous polarisation of both beams can significantly expand the accessible physics opportunities compared to the case of $e^{-}$polarisation only ${ }^{4}$. The use of simultaneously polarised $e^{-}, e^{+}$beams has several advantages for: determining quantum numbers of new particles, providing higher sensitivity to non-standard couplings, increasing rates and background suppression, raising the effective luminosity and expanding the range of measurable experimental observables e.g. with the help of transversely polarised beams.

The author would like to thank Yousef Makdisi with his nice and friendly organising team for a wonderful and very interesting conference! G.M.-P. was partially supported by BNL.

\footnotetext{
${ }^{4}$ For updates see POWER group (Polarisation at Work in Energetic Reactions) http://www.ippp.dur.ac.uk/ gudrid/power/
} 

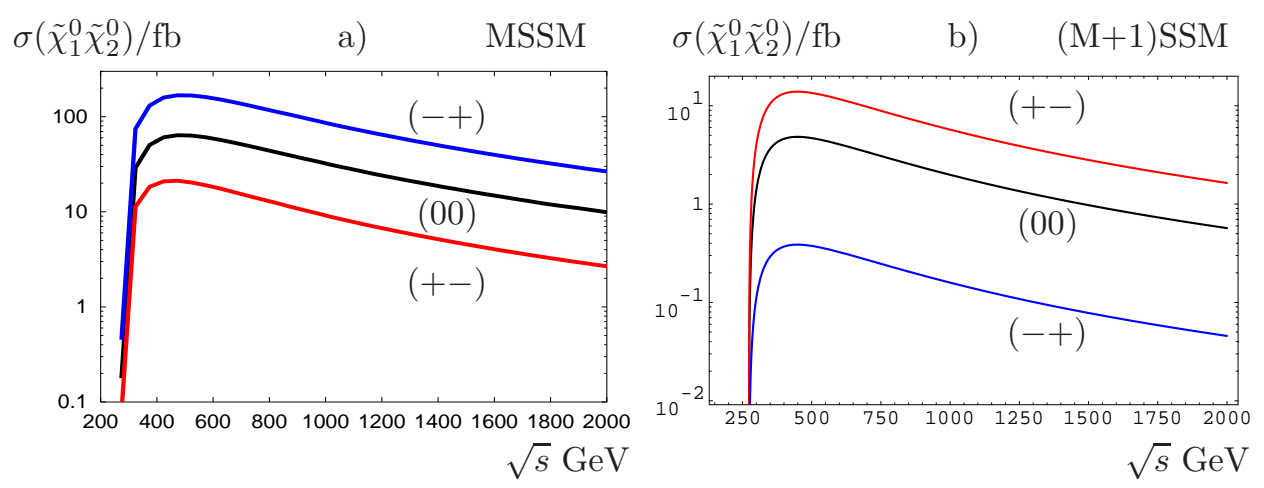

Figure 5: Cross sections for the process $\sigma\left(e^{+} e^{-} \rightarrow \tilde{\chi}_{1}^{0} \tilde{\chi}_{2}^{0}\right)$ with polarised beams $\left(P_{e^{-}}= \pm 80 \%, P_{e^{+}}=\mp 60 \%\right)$ for an example in a) the MSSM and in $b)$ the $(\mathrm{M}+1) \mathrm{SSM}$, where the mass spectra of the light neutralinos are similar 23].

\section{References}

[1] J. A. Aguilar-Saavedra et al., ECFA/DESY LC Physics Working Group Collaboration, hep-ph/0106315.

[2] LHC/LC working group, see http://www.ippp.dur.ac.uk/georg/lhclc/, contact persons: Georg Weiglein, Rohini Godbole, Frank Paige.

[3] G. Moortgat-Pick and H. M. Steiner, Eur. Phys. J. directC 6 (2001) 1 hep-ph/0106155; J. Erler et al., in Proc. of the APS/DPF/DPB Summer Study on the Future of Particle Physics (Snowmass 2001) ed. N. Graf, hep-ph/0112070; G. Moortgat-Pick et al., hep-ph/0210212.

[4] T. Abe et al., American Linear Collider Working Group Collaboration, in Proc. of the APS/DPF/DPB Summer Study on the Future of Particle Physics (Snowmass 2001) ed. N. Graf, SLAC-R-570 Resource book for Snowmass 2001, 30 Jun - 21 Jul 2001, Snowmass, Colorado, hep-ex/0106056, hep-ex/0106057.

[5] V.E. Balakin and A.A. Mikhailichenko, INP 79-85 (1979); K. Flöttmann, DESY-93-161, 1993; K. Flöttmann, DESY-95-064, 1995.

[6] see Talk by John Sheppard on the POWER meeting, June 8-9, 2002, Durham: http://www.desy.de//gudrid/power/schedule_power_fin.html and also http://www.slac.stanford.edu/ achim/positrons/. 
[7] V. Gharibyan, N. Meyners, K.P. Schüler, LC-DET-2001-047.

[8] G. Alexander and I. Cohen, Nucl. Instrum. Meth. A 486 (2002) 552, hep-ex/0006007.

[9] A. Blondel, Phys. Lett. B202 (1988) 145.

[10] see also talk by Klaus Desch, International Workshop on Linear Colliders August 26-30, 2002 Jeju Island, Korea: http://lcws2002.korea.ac.kr/

[11] R. Hawkings and K. Moenig, EPJdirect C 8 (1999) 1; K. Mönig, LCPHSM-2000-059; W. Menges, LC-PHSM-2001-022.

[12] J. Fleischer, K. Kolodziej and F. Jegerlehner, Phys. Rev. D 49 (1994) 2174.

[13] J. Erler, S. Heinemeyer, W. Hollik, G. Weiglein and P. M. Zerwas, Phys. Lett. B 486 (2000) 125, hep-ph/0005024.

[14] B. Ananthanarayan, S. D. Rindani and A. Stahl, LC-PHSM-2002-006, hep-ph/0204233.

[15] A. Leike, S. Riemann, Z. Phys.C75 (1997) 341; S. Riemann, hep-ph/9610513, Proceedings of the 1996 DPF / DPB Summer Study on New Directions for High-energy Physics; R. Casalbuoni, S. De Curtis, D. Dominici, R. Gatto, S. Riemann, LC-TH-2000-006 hep-ph/0001215 ; S. Riemann, Proceedings of 4th International Workshop on Linear Colliders (LCWS 99), Sitges, Barcelona, Spain; S. Riemann, LC-TH-2001-007; A. A. Pankov and N. Paver, hep-ph/0209058.

[16] A. Bartl, H. Eberl, S. Kraml, W. Majerotto, W. Porod and A. Sopczak, Z. Phys. C 76 (1997) 549, hep-ph/9701336; A. Bartl, H. Eberl, S. Kraml, W. Majerotto, W. Porod and A. Sopczak, hep-ph/9604221; A. Bartl, H. Eberl, S. Kraml, W. Majerotto and W. Porod, Z. Phys. C 73 (1997) 469 hep-ph/9603410; A. Bartl, K. Hidaka, T. Kernreiter and W. Porod, hep-ph/0207186 and Phys. Lett. B 538 (2002) 137, hep-ph/0204071;

[17] M. M. Nojiri, Phys. Rev. D 51 (1995) 6281, hep-ph/9412374; M. M. Nojiri, K. Fujii and T. Tsukamoto, Phys. Rev. D 54 (1996) 6756, hep-ph/9606370; M. Guchait and D. P. Roy, Phys. Lett. B 535 (2002) 243, hep-ph/0205015. 
[18] C. Blöchinger, H. Fraas, G. Moortgat-Pick and W. Porod, Eur. Phys. J. C 24 (2002) 297, hep-ph/0201282.

[19] S. Y. Choi, J. Kalinowski, G. Moortgat-Pick and P. M. Zerwas, Eur. Phys. J. C 22 (2001) 563, hep-ph/0108117; S. Y. Choi, J. Kalinowski, G. Moortgat-Pick and P. M. Zerwas, Eur. Phys. J. C 23 (2002) 769, hep-ph/0202039.

[20] J. F. Gunion, T. Han, J. Jiang, S. Mrenna and A. Sopczak, in Proc. of the APS/DPF/DPB Summer Study on the Future of Particle Physics (Snowmass 2001) ed. N. Graf, hep-ph/0112334; V. D. Barger, T. Han and J. Jiang, Phys. Rev. D 63 (2001) 075002, hep-ph/0006223; J. L. Feng and T. Moroi, Phys. Rev. D 56 (1997) 5962, hep-ph/9612333.

[21] E. Boos, G. Moortgat-Pick, H. U. Martyn, M. Sachwitz and A. Vologdin, hep-ph/0211040; E. Boos, U. Martyn, G. Moortgat-Pick, M. Sachwitz, A. Sherstnev, P.M. Zerwas, hep-ph/0303110.

[22] M. Heyssler, R. Rückl, H. Spiesberger, Proceedings of the LCWorkshop, Sitges 1999; Private communication with H. Spiesberger.

[23] S. Hesselbach, F. Franke, H. Fraas, hep-ph/0003272; F. Franke and S. Hesselbach, Phys. Lett. B 526 (2002) 370, hep-ph/0111285; G. Moortgat-Pick, S. Hesselbach, F. Franke and H. Fraas, hep-ph/9909549.

[24] A. Vest, LC-TH-2000-058. G. Wilson, LC-PHSM-2001-010. 